\title{
Trends in Mitochondrial Therapeutics for Neurological Disease
}

\author{
Ana Leitão-Rocha, Pedro Guedes-Dias, Brígida R. Pinho, Jorge M. A. Oliveira ${ }^{\bowtie}$ \\ REQUIMTE, Department of Drug Sciences, Faculty of Pharmacy, University of Porto, \\ Portugal (两jorgemao@ff.up.pt)
}

\begin{abstract}
Neuronal homeostasis is critically dependent on healthy mitochondria. Mutations in mitochondrial DNA (mtDNA), in nuclear-encoded mitochondrial components, and agedependent mitochondrial damage, have all been connected with neurological disorders. These include not only typical mitochondrial syndromes with neurological features such as encephalomyopathy, myoclonic epilepsy, neuropathy and ataxia; but also secondary mitochondrial involvement in neurodegenerative disorders such as Alzheimer's, Parkinson's and Huntington's disease. Unravelling the molecular aetiology of mitochondrial dysfunction opens new therapeutic prospects for diseases thus far lacking effective treatments. In this review we address recent advances on preventive strategies, such as pronuclear, spindlechromosome complex, or polar body genome transfer to replace mtDNA and avoid disease transmission to newborns; we also address experimental mitochondrial therapeutics aiming to benefit symptomatic patients and prevent disease manifestation in those at risk. Specifically, we focus on: (1) gene therapy to reduce mutant mtDNA, such as anti-replicative therapies and mitochondria-targeted nucleases allowing favourable heteroplasmic shifts; (2) allotopic expression of recoded wild-type mitochondrial genes, including targeted tRNAs and xenotopic expression of cognate genes to compensate for pathogenic mutations; (3) mitochondria targeted-peptides and lipophilic cations for in vivo delivery of antioxidants or other putative therapeutics; and (4) modulation of mitochondrial dynamics at the level of biogenesis, fission, fusion, movement and mitophagy. Further advances in therapeutic development are hindered by scarce in vivo models for mitochondrial disease, with the bulk of available data coming from cellular models. Nevertheless, wherever available, we also address data from in vivo experiments and clinical trials, focusing on neurological disease models.
\end{abstract}

\section{INTRODUCTION}

Neuronal physiology is intricately linked with mitochondrial bioenergetics and dynamics. It comes therefore at no surprise that mitochondrial failure eventually leads to neuronal demise. Mitochondrial dysfunction may in fact affect multiple organs or tissues, but the energetic needs and the non-dividing nature of neurons place the nervous system at particularly high risk. Neuronal ion balance continuously demands ATP from an active mitochondrial respiratory chain [1], which integrates five multi-subunit complexes embedded in the inner mitochondrial membrane (IMM). With the exception of complex II, fully encoded by nuclear DNA (nDNA), all other respiratory complexes have a dual genetic origin, with some subunits encoded by nDNA and others by mitochondrial DNA (mtDNA). Respiratory complexes require nDNA-encoded factors for proper assembly and addition of prosthetic groups. In fact, the nucleus encodes all proteins involved in mtDNA replication, transcription, translation and repair, as well as mt-tRNA synthetases, mitochondrial ribosomal proteins, and proteins involved in maintaining the mitochondrial deoxynucleoside triphosphate pool [2]. Multiple Mendelian-inherited nDNA mutations may thus cause respiratory chain dysfunction. 
Primary mitochondrial dysfunction also arises from mutations in mtDNA, which encodes 13 respiratory chain subunits plus 2 rRNAS and 22 tRNAs required for intra-mitochondrial protein synthesis. Specific features of mitochondrial genetics include the (practically) exclusive maternal inheritance of multiple mtDNA copies, which can be identical (homoplasmy) or different within the same cell (heteroplasmy). Also, random mitotic segregation of mtDNA, variable heteroplasmy, potential for clonal expansion, and diverse mutational thresholds, condition the differential expression of biochemical defects across cells and tissues [3]. Such idiosyncratic features contribute for a wide variability of mitochondrial disease manifestation and for the complex clinical maze of mitochondrial neurology [4].

Neurological symptoms accompany several diseases resulting from mtDNA mutations, namely, mitochondrial encephalomyopathy, lactic acidosis and stroke-like episodes (MELAS); myoclonic epilepsy and ragged red fibres (MERRF); neuropathy, ataxia, and retinitis pigmentosa (NARP); Leber's hereditary optic neuropathy (LHON); and maternally inherited Leigh syndrome, among others [5]. It is instructive that complex I deficiency in Leigh syndrome may stem not only from mutations in mtDNA but also in the nDNA-encoded subunits and assembly factors [2]. In fact, even when mtDNA is free from pathogenic mutations and all nuclear-encoded respiratory complex subunits are correctly expressed; multiple 'indirect hits' can still cause mitochondrial-associated neurological disease. Mutations in respiratory chain ancillary proteins are key examples of such indirect hits. Also, mutations in the mt-RNA translation machinery, in enzymes governing the IMM lipid milieu, and in coordinators of the mitochondrial fission-fusion equilibrium are increasingly identified as causes for neurological disorders [4]. The latter are typically illustrated by mutations in the pro-fusion proteins OPA1 and Mfn2, respectively associated with dominant optic atrophy and Charcot-Marie-Tooth type 2A neuropathy. Loss of function mutations in PINK1 and Parkin, causing hereditary Parkinson's disease (PD), provide another key link between abnormal mitochondrial dynamics, in this case mitophagy, and neurodegeneration. Even when mitochondrial damage is a secondary pathological event, it still plays a prominent role in key neurodegenerative disorders such as Alzheimer's disease (AD), Huntington's disease (HD) and spinocerebellar ataxias, amyotrophic lateral sclerosis, and several others [6, 7]. Interestingly, differences in mitochondrial physiology across brain regions may contribute, together with other cellular and environmental factors, for differential vulnerability patterns to neurological disease $[8,9]$.

Experimental models of mitochondrial disease are primarily cell-based. Cytoplasmic hybrid cells (cybrids; generated by depleting host cells from endogenous mtDNA and repopulating with mtDNA from patient cells), are widely used to address the relative contribution of nuclear vs. mitochondrial backgrounds for a given disease [10]. The yeast Saccharomyces cerevisae is also widely used due to the feasibility of mitochondrial genetic transformation in this organism [11]. Still, neither yeast nor cybrids model neuron-specific traits, much less model the complex features of a whole organism. To address these issues, mouse embryonic stem cells cybrids containing mtDNA mutation have been differentiated into neurons [12], and multiple techniques are available for the study of mitochondrial function in live neurons [13]. Mitochondrial disease caused by some nDNA mutations has already been modelled in mice by nuclear transgenesis, but mtDNA mutations are more challenging to model in animals [14]. Still, progresses are being made in the development of mouse models of mtDNA defects [15], and models of mitochondrial disease are also being developed in zebrafish, drosophila and Caenorhabditis elegans [16-19].

A systematic review of clinical data on mitochondrial therapeutics revealed that although statistically significant clinical improvements are reported in low-quality studies, they were not confirmed by subsequent high-quality studies. Currently prescribed drugs, consisting mostly of vitamin supplements, metabolic precursors and antioxidants lack convincing 
The published manuscript is available at EurekaSelect via http://www.eurekaselect.com/128349/article

evidence for efficacy other than that expected for placebo. Further, 'symptomatic improvements' due to normal growth and development (in the case of affected children), and regression to mean symptoms (in diseases where symptoms randomly fluctuate), are not always adequately controlled for in available trials [20]. There is therefore a great need for persistent improvement in clinical trial design, identification of reliable biomarkers, and further basic research on disease mechanisms and experimental therapeutics. In this review, we address recent advances on preventive strategies associated with assisted reproduction techniques, such as pronuclear, spindle-chromosome complex, or polar body genome transfer. We also address experimental mitochondrial therapeutics aiming to benefit symptomatic patients and prevent disease manifestation in those at risk. We focus on advances in different modalities of gene therapy, mitochondria-targeted drugs, and modulators of mitochondrial dynamics. The bulk of current data comes from cell-based experiments, nevertheless, wherever available, we address data from in vivo experiments and clinical trials focusing on neurological disease models.

\section{MITOCHONDRIAL THERAPEUTIC STRATEGIES}

\subsection{Preventing transmission by mitochondrial gene replacement}

Assisted conception strategies have the potential to prevent or at least reduce the risk of transmitting mitochondrial disease. Afflicted families can benefit from genetic counseling, but this is particularly challenging and associated with high level of uncertainty in the case of mtDNA mutations [21]. Pre-implantation genetic diagnosis (PGD) can assist selection of embryos without disease-linked nuclear DNA mutations. Mutations in mtDNA, however, are solely maternally inherited and may in principle be passed to all offspring, albeit in uncertain proportion. PGD to select embryos with very low mutant mtDNA levels reduces risk but does not eliminate the possibility that mitochondrial disease might manifest, or that asymptomatic girls will be carriers in their oocytes. Moreover, PGD is not helpful when homoplasmic mutations or high levels of heteroplasmic mtDNA mutations are already present in oocytes [22]. Development of other options for preventing transmission of mtDNA disease has thus been actively explored, primarily nuclear genome transplantation into the healthy mtDNA background of donor zygotes/oocytes via pronuclear or spindle transfer techniques [23, 24].

Pronuclear transfer into enucleated zygotes has been successfully applied in mice with pathogenic mtDNA mutations, yielding an $\mathrm{F}_{0}$ progeny free of respiration defects throughout their lives [25]. Proof of principle experiments with the same technique were conducted in abnormally fertilized human zygotes, achieving less than $2 \%$ carryover of mtDNA and thus supporting its potential to prevent mtDNA disease transmission in humans [26]. Another technique for mtDNA replacement, spindle-chromosome complex transfer, has been successfully applied to non-human primates generating healthy offspring without detectable spindle-donor mtDNA [27]. A recently-published 3-year follow up on those primates has shown that they continue to develop normally, with comparable growth and metabolic status to age-matched controls, and without significant changes in mtDNA carryover and heteroplasmy in blood and skin samples [28]. In the same publication, authors demonstrated the feasibility of replacing mtDNA in human oocytes via spindle transfer. Normally fertilized human oocytes developed comparably to controls into blastocysts, and the derived embryonic stem cell lines exhibited normal euploid karyotypes, with undetectable carryover mtDNA [28]. Still, the current frequency of premature activation/abnormal fertilization of human oocytes with the spindle transfer technique is far from ideal [28], and methodological changes have been recently proposed [29]. In spite of major and encouraging advances, further 
efficacy and safety studies are required before either pronuclear or spindle transfer techniques can be introduced into clinical practice ([30]; see also the 2014 update of the UK's Human Fertilisation and Embryology Authority report at: www.hfea.gov.uk/6372.html). As recently cautioned, however, pronuclear and spindle transfer may only benefit a small group of female mtDNA mutation carriers, whilst prenatal diagnostic testing can be used for all Mendelian mitochondrial disorders and for the majority of mtDNA mutations [31].

Polar body genome transfer has been recently proposed as an alternative technique for mtDNA replacement. This procedure, so far tested in mice, consists in transferring the genetic information of a polar body from a donor oocyte into a recipient oocyte with normal mitochondria [32]. Key advantages are that fewer mitochondria are carried over during transfer and, combined with spindle transfer, a single oocyte could offer two donor genome sources for a mtDNA replacement procedure, reducing the number of necessary donor oocytes [33]. Evidently, while all these techniques may prove useful for preventing mitochondrial disease transmission to newborn children, different approaches must be developed in order to treat symptomatic patients and to prevent disease in those already at risk [34].

\subsection{Gene therapy to reduce or compensate for mutant mtDNA}

Pathogenic mtDNA mutations are most often heteroplasmic, meaning that they coexist with wild-type mtDNA. Some gene therapy strategies thus aim to shift the balance towards wildtype mtDNA by reducing mutant mtDNA below the disease threshold. These include antigenomic approaches to halt replication of mutant mtDNA or mitochondrial-targeted nucleases to selectively degrade mutated mtDNA [35]. Other strategies compensate for mutant mtDNA via allotopic expression of the wild-type genes, or via xenotopic expression of cognate genes from other species [36]. Proof of principle in cellular models has been established for most of these strategies, but there is still very limited in vivo evidence for their safety and efficacy. Problems with delivery of the therapeutic agents to affected tissues remain main hindrances to drug development, together with the scarcity of good animal models for pre-clinical testing. Key strategies and their recent advances are summarized below.

Antigenomic (or anti-replicative) therapies have been tested with sequence-specific nucleic acid derivatives, designed to bind mutant mtDNA and inhibit its replication, whilst allowing wild-type mtDNA propagation. Peptide nucleic acids (PNAs) showed promising in vitro results [37], but PNAs have so far failed to modulate heteroplasmy in intact cells, as a result of their inability to cross the IMM and access mtDNA [35]. Recently, RNA vectors based on $5 \mathrm{~S}$ rRNA or tRNA were reported capable of delivering anti-replicative oligoribonucleotides to mitochondria by exploring RNA import pathways. The same study reported a transient 15$35 \%$ decrease in mutant mtDNA levels in Kearns Sayre syndrome cell models using such anti-replicative RNAs [38]. Attempts to increase these agents lifetime by chemical modification of the oligonucleotides have so far resulted in loss of efficacy [39, 40].

Restriction endonucleases fused to a mitochondria-targeting (mito-) sequence were shown to selectively reduce mtDNA containing the recognized restriction site [41]. Experiments with a heteroplasmic cell line showed that mito-SmaI selectively depleted pathogenic m8993T $>\mathrm{G}$ mtDNA allowing for repopulation with wild-type mtDNA [42]. In vivo experiments with NZB/BALB heteroplasmic mice showed that adenoviral vectors injected in brain and muscle efficiently delivered mito-ApaLI, which selectively degraded BALB mtDNA [43]. Systemic delivery of mito-ApaLI via cardiotropic (AAV6) or hepatotropic (Ad5) adeno-associated virus increased NZB mtDNA in target tissues [44], whereas AAV9-mediated delivery of 
mito-ApaLI to newborn mice reduced mtDNA heteroplasmy in all striated muscles, including the heart [45]. Endonuclease-mediated heteroplasmy shift is thus rapidly emerging as one of the most promising therapeutic approaches for mtDNA mutations. While the use of bacterial restriction nucleases requires a unique site in mutated mtDNA (but see the 'differential multiple cleavage site model'; [46]), other nucleases are more versatile. Zinc-finger nucleases (ZFNs) can be engineered to bind a specific DNA sequence, and mito-ZFNs were shown to selectively degrade mutated mtDNA in heteroplasmic cells [47, 48]. The subsequently developed transcription activator-like effector nucleases (TALENs) are an interesting alternative to ZFNs [49]. Transient mito-TALENs expression in patient-derived cells reduced deletion or point-mutant mtDNA, fueling the expectation that a few mito-TALEN administrations may suffice to correct heteroplasmy and rescue oxidative phosphorylation defects [50]. Recently, novel obligatory heterodimeric mito-ZFNs have shown improved heteroplasmy shifting and rescue of mitochondrial respiration, meeting the efficiency of mitoTALENs, with the advantage of being smaller constructs and thus more amenable to packaging in viral vectors [51]. Further development of these strategies, namely in vivo studies, are currently hindered by the size of the constructs, and challenged by the risk of severe mtDNA depletion, particularly in tissues containing high proportions of the targeted mutant mtDNA [34, 52].

Allotopic expression relies on nuclear-cytosolic synthesis of a recoded wild-type copy of the mutated mtDNA gene, plus its functional incorporation into mitochondria by means of a removable targeting signal. Feasibility was demonstrated in yeast, where cytoplasmically synthesized subunit 8 was successfully integrated into the mitochondrial ATPase complex [53]. Attempts in mammalian cells yielded contradictory results, and thus far lack unequivocal demonstration that the allotopically-expressed subunits assemble into the respective holoenzymes and are responsible for improved function [54]. Cytoplasmically synthesized ATPase 6 was reported to incorporate into mitochondria and rescue defective ATP synthesis in human cell lines with the m8993 $>\mathrm{G}$ mutation $[55,56]$. Independent reformulation of these experiments, however, found neither evidence for ATPase 6 incorporation into the ATP synthase holoenzyme, nor for improvement in mitochondrial function, suggesting that earlier studies might have been biased by random clonal variation in ATP synthesis within the aneuploid cell lines [57]. Similarly, successful allotopic expression of the ND4 subunit to rescue the m11778G>A LHON mutation in human cells [58] was contradicted by subsequent studies [59]. Experiments with mtDNA-encoded genes in mammalian cells showed that ATPase 8 could be allotopicaly expressed and localized to mitochondria, whereas the more hydrophobic ND4 and apocytochrome $\mathrm{b}$ formed aggregates and induced loss of mitochondrial membrane potential [59].

To overcome hidrophobicity issues, 3'UTR sequences were engineered into the mRNAs to target them near mitochondria and optimize co-translational translocation of the allotopicallyexpressed gene. This optimized strategy was reported successful for the allotopic expression of ND1, ND4 and ATP6 to rescue mtDNA mutations affecting complex I and V in patients fibroblasts $[60,61]$. Also, optimized allotopic expression of human ND4 was reported to prevent blindness in rats whose eyes were previously electroporated with human ND4 harboring the $\mathrm{m} 11778 \mathrm{G}>\mathrm{A}$ LHON mutation [62]. Clinical trial recruitment of LHON patients carrying the $\mathrm{m} 11778 \mathrm{G}>\mathrm{A}$ mutation has started [63]. The trial identified as NCT01267422 reports the random treatment of seven patients by single intravitreal injection of recombinant AAV2-ND4 (Clinicaltrials.gov, last processed on August 24, 2014). While some recent studies are supportive of the importance and strategies of these ND4 expression trials [64] [65], other studies caution that: (i) available data on allotopic expression is insufficient to initiate clinical trials in humans; (ii) reports of successful allotopic expression by phenotypic recovery in cells with mtDNA mutations may be explained by selection of revertants, without 
mitochondrial internalization and/or holoenzyme incorporation of the expressed subunit; (iii) the conceptual validity of the mouse LHON model is questioned given the use of human mtDNA sequences, plus the possibility that ND4 hydrophobicity causes retinal toxicity by collapsing the mitochondrial import machinery [54].

Targeted tRNAs have been tested as a strategy to overcome mutations in mitochondrial tRNA (mt-tRNA) genes. Yeast tRNA ${ }^{\text {Lys }}$ derivatives expressed in patient fibroblasts with the m8344A $>$ G MERRF mutation were imported to mitochondria, correctly aminoacylated, and shown capable of rescuing mitochondrial translation and respiratory chain activity [66]. Interestingly, tRNA import appears more widespread than previously thought, with both rat and human mitochondria being capable of importing nucleus-encoded $\operatorname{tRNA}^{\mathrm{Gln}(\mathrm{CUG})}$ and tRNA $^{\mathrm{Gln}(\mathrm{UUG})}$ in vivo, by a distinct mechanism from protein import [67]. A wide range of tRNAs and mRNAs may be targeted to mitochondria by appending the RNA component of human RNase P, which interacts with polynucleotine phosphorylase (PNPase) that facilitates delivery of the hybrid RNA into the mitochondrial matrix. Further appending a 3'-UTR localization sequence, however, was essential for efficient translocation of corrective mttRNAs, shown capable of rescuing mitochondrial translation and respiratory defects in two human cell lines with mutant mt-tRNA genes [68].

Overexpression of aminoacyl tRNA synthetases is an alternative strategy to rescue cognate pathogenic mt-tRNA mutations and, more recently, non-cognate mutations [69]). Accordingly, modification of the mt-tRNA binding domain of human mitochondrial phenylalanyl-tRNA synthetase significantly improved the aminoacylation efficiency of mttRNA $^{\text {Phe }}$ carrying the $\mathrm{m} 611 \mathrm{G}>\mathrm{A}$ MERRF mutation [70]. Overexpression of human mitochondrial valyl- or leucyl-tRNA synthetases increased, respectively, the steady-state levels of mutant mt-tRNA ${ }^{\mathrm{Val}}$ [71] or mt-tRNA ${ }^{\mathrm{Leu}(\mathrm{UUR})}$ [72], consistent with increased stability of the charged mt-tRNA. Also, overexpression of mitochondrial leucyl-tRNA synthetase rescued mitochondrial translation and respiration in cells carrying the A3243G MELAS mutation in mt-tRNA ${ }^{\text {Leu(UUR) }}$ [73]. A related strategy consists in the allotopic expression of recombinant and importable tRNAs with altered aminoacylation identity. Yeast tRNAs with artificially changed identity, from cytoplasmic lysine to mitochondrial leucine, partially rescued mitochondrial translation, steady-state levels of mtDNA-encoded respiratory chain subunits, and cellular respiration in human cells with the A3243G MELAS mutation in mttRNA $^{\text {Leu }}$ [74]. More recently, human mitochondrial leucyl tRNA synthetase, particularly a carboxy terminal fragment, was reported to rescue respiratory deficiency induced by pathogenic mt-tRNA mutations [69, 75]. Mechanisms, particularly those involved in the suppression of non-cognate mutations, remain uncertain but unlikely to involve aminoacylation (domain absent from the carboxy terminal fragment), and are possibly related to stabilization of the mutant tRNAs. These findings raise the appealing possibility of developing a universal suppressor molecule for multiple mt-tRNA mutations [76].

Xenotopic expression of non-mammalian genes that can function as single respiratory chain subunits provides an encouraging alternative to allotopic expression. The rationale is that functional single subunits would obviate the need for integration into assembled multiprotein complexes, which has been the crux of allotopically expressed mammalian subunits. The Ciona intestinalis single subunit alternative oxidase (AOX) or the Saccharomyces cerevisae single subunit NADH oxidase (Ndi1) have the potential to alleviate, respectively, deficiencies in cytochrome $c$ oxidase (COX) or in complex I [36]. C. intestinalis AOX expression in human cells conferred cyanide resistance to mitochondrial substrate oxidation [77], and corrected decreased cell respiration, glucose and pyruvate dependency in COX15deficient fibroblasts derived from a patient with early fatal cardiomyopathy [78]. Xenotopically expressed AOX integrates the mammalian mitochondrial respiratory chain where it can transfer electrons from the reduced ubiquinone pool to oxygen, thus bypassing 
downstream blockades. Unlike downstream complex III and IV, however, AOX is not a proton pump, and thus it is crucial to establish whether the advantages of a potential superoxide-limiting bypass outweigh the disadvantages of energy dissipation. AOX was safely expressed in mice (MitAOX) without obvious detrimental effects upon major physiological parameters, which suggests that AOX does not severely limit energy production when integrated in an otherwise normal respiratory chain. The increased survival of MitAOX mice exposed to lethal cyanide supports the possibility that AOX may counteract downstream respiratory chain impairment [79]. MitAOX mice are thus a promising tool for further evaluation of the therapeutic potential of AOX in mitochondrial disease.

Expression of yeast Ndil restored NADH dehydrogenase activity in human cells lacking complex I activity due to ND4 gene mutation [80]. Ndi1 insensitivity to complex I inhibitors such as rotenone and 1-methyl-4-phenyl-1,2,3,6-tetrahydropyridine (MPTP) promoted its testing in PD or LHON models induced with such compounds. Viral delivery of Ndi1 (rAAVNDI1) by unilateral injection to the substantia nigra protected mice from neuronal injury induced by either MPTP [81] or rotenone [82], providing in vivo evidence for functional complementation of complex I by Ndil. Also, long term Ndil expression ( $>$ 8months) was neuroprotective in a chronic (1-4 weeks) MPTP/probenecid mouse model [83]. Ndi1 expression was also protective in rotenone-induced mouse models of mitochondrial optic neuropathy, preserving retinal function and ameliorating vision [84, 85]. Concerning in vivo genetic models, a study with drosophila PINK1/Parkin mutants showed that Ndi1 rescued PINK1-associated defects, but failed to rescue any of the Parkin mutant phenotypes. Significantly, PINK1 mutants exhibited decreased complex I activity, unlike Parkin mutants [86]. Further in vivo studies including mammalian genetic models of mitochondrial disease would help define the therapeutic potential of xenotopic expression.

\subsection{Lipophilic cations and mitochondria-targeted peptides}

Lipophilic cations and mitochondria-targeted peptides are important agents for in vivo drug delivery, allowing enhanced mitochondrial accumulation of otherwise poorly taken up molecules. These delivery strategies increase potency of the targeted compounds, decreasing the required dose as well as extramitochondrial metabolism, inactivation and side effects. These strategies have two important limitations: (i) they lack organ-specificity, leading to preferential accumulation in mitochondria-rich tissues; and (ii) the typically used chemicals tend to accumulate in the matrix and the matrix-facing surface of the IMM, by comparison with other important mitochondrial compartments [87].

Mitochondrial membrane potential $\left(\Delta \psi_{\mathrm{m}}\right)$ drives accumulation of lipophylic cations such as triphenylphosphonium $\left(\mathrm{TPP}^{+}\right)$derivatives, whose concentration within mitochondria increases approximately 10 -fold for every $60 \mathrm{mV}$ of $\Delta \psi_{\mathrm{m}}$ as described by the Nernst equation. The $\mathrm{TPP}^{+}$-modified ubiquinone, MitoQ, has been extensively investigated as a mitochondria targeted-antioxidant, and shown protective in multiple disease models [88]. Oral MitoQ improved survival and grip strength in the SOD1 ${ }^{\mathrm{G} 93 \mathrm{~A}}$ mouse model of amyotrophic lateral sclerosis, in correlation with slower decline of mitochondrial respiration, reducing oxidative markers, astrogliolis and neuronal loss in the spinal cord [89]. MitoQ was also reported protective in rodent models of MPTP-induced PD [88]. A clinical trial in 128 patients treated for 12 month with MitoQ, however, failed to show any difference versus placebo in PD progression (NCT00329056 at Clinicaltrials.gov; [90]). Still, MitoQ was well tolerated and it remains to be ascertained whether early pre-symptomatic treatment, preceding overt dopaminergic degeneration, might prove beneficial in delaying the onset of PD. 
The published manuscript is available at EurekaSelect via http://www.eurekaselect.com/128349/article

Szeto-Schiller (SS) peptides are described to selectively target mitochondria and concentrate in the IMM, independently of $\Delta \psi_{\mathrm{m}}$, by yet uncertain mechanisms (but see [91]) even though the peptides contain a net positive charge at physiological $\mathrm{pH}$ [92]. Potentialindependent mitochondrial uptake would offer a significant advantage since dysfunctional mitochondria are likely to exhibit lower $\Delta \psi_{\mathrm{m}}$, thus excluding or limiting the uptake of $\Delta \psi_{\mathrm{m}}-$ dependent drugs such as lipophilic cations. SS peptides display antioxidant activity, with SS31 exerting protective effects in animal models of amyotrophic lateral sclerosis [93], PD [94], and in primary neurons derived from AD mice - an in vitro model where MitoQ was also found protective [95]. SS-31 was also reported to improve mitochondrial energetics and muscle performance in aged mice [96]. The ongoing and future clinical trials with a modified form of SS-31 [92] should help establish its therapeutic potential.

Hemigramicidin-TEMPO conjugates are antioxidants targeted to mitochondria via peptides derived from the membrane-active antibiotic, gramicidin $\mathrm{S}$, which carry the stable free radical 4-amino-2,2,6,6-tetramethylpiperidine- $N$-oxyl (4- $\mathrm{NH}_{2}$-TEMPO) as a ROS scavenger [97]. XJB-5-131 is one of the most promising compounds in this class. The mitochondrial targeting moiety is an alkene peptide isostere modification of the Leu-D-Phe-Pro-Val-Orn gramicidin S segment, whose $\beta$-turn motif inserts into the mitochondrial membrane. This mitochondrial targeting strategy is $\Delta \psi_{\mathrm{m}}$-independent, avoiding exclusion of XJB-5-131 from depolarized mitochondria. XJB-5-131 was recently reported to enhance survival of primary neurons, reduce oxidative damage to mtDNA, improve mitochondrial function, and suppress motor decline in a HD mouse model [98].

Peptide-based-multi-walled carbon nanotubes are being developed as an alternative delivery system for therapeutic oligonucleotides against mtDNA mutations. Mitochondrialtargeting of these nanotubes is promoted by functionalization with a 25 amino acid peptide derived from the N-terminal region of human COX subunit VIII [99]. While these carbon nanotubes can clearly be internalized by cultured phagocytes and colocalize with their mitochondria, the in vivo pharmacokinetics and the bioenergetic consequences of inserting possibly $\Delta \psi_{\mathrm{m}}$-collapsing tubes into mitochondrial membranes remain to be investigated.

\subsection{Modulation of mitochondrial dynamics}

\subsubsection{Biogenesis and fission-fusion}

The induction of mitochondrial biogenesis is being actively explored as a strategy to overcome mitochondrial dysfunction. The peroxisome proliferator-activated receptor (PPAR) and its co-activator $\alpha(\mathrm{PGC}-1 \alpha)$ play key regulatory roles in biogenesis activation, highlighting the PPAR/PGC-1 pathway as a druggable target. Transgenic expression of PGC$1 \alpha$ or treatment with the PPAR agonist bezafibrate induced mitochondrial biogenesis in vivo, preventing mitochondrial myopathy in mice lacking an essential COX assembly factor in skeletal muscle [100]. Bezafibrate, however, failed to induce mitochondrial biogenesis in other COX deficient models [101], in contrast with PGC-1 $\alpha$ overexpression or its indirect activation with the AMPK agonist AICAR [102]. Still, bezafibrate restored PGC-1 $\alpha$ levels, induced mitochondrial biogenesis, and exerted neuroprotective effects in mouse models of either HD [103] or tau pathology [104]. Moreover, bezafibrate increased mitochondrial proteins and ATP generating capacity, being neuroprotective in a mouse model of mitochondrial encephalopathy [105]. Thus, whilst there is conflicting data on bezafibrate effects in skeletal muscle, data from neurological disease models suggest that it might be a promising compound for the treatment of mitochondrial-associated neurodegenerative disease. 
PPAR- $\gamma$ agonists, such as the thiazolidinediones rosiglitazone and pioglitazone, exhibited promising effects in pre-clinical models of neurodegeneration, but data from clinical trials are unsupportive of their use in AD patients [106], and their potential in the context of PD is still uncertain [107]. A tentative pre-clinical evaluation of the bioenergetic intermediate oxaloacetate, as potential treatment for $\mathrm{AD}$ and other neurodegenerative disorders, reported increased PGC-1 $\alpha$ mRNA, activated mitochondrial biogenesis and hippocampal neurogenesis in $\mathrm{C} 57 \mathrm{Bl} / 6$ mice [108].

Lysine deacetylases (KDACs), including the $\mathrm{NAD}^{+}$-dependent sirtuins, are reported to regulate mitochondrial biogenesis via modulation of PGC- $1 \alpha$ activity and changes in gene expression, suggesting that KDAC modulatory drugs may provide a strategy to boost mitochondrial biogenesis $[109,110]$. Increasing $\mathrm{NAD}^{+}$availability through administration of the precursor nicotinamide riboside was reported to increase PGC- $1 \alpha$-dependent mitochondrial biogenesis, presenting beneficial effect in the Tg2576 AD mouse model [111]. Recently, nicotinamide riboside or MRLB-45696 (an inhibitor of the NAD ${ }^{+}$consuming enzyme poly(ADP-ribose) polymerase - PARP1) were reported to increase mitochondrial biogenesis with promising results in mitochondrial myopathy models [112-114]. Still, further studies on dosage regimens and pharmacokinetic data are still required for a more detailed evaluation of the therapeutic potential of these $\mathrm{NAD}^{+}$modulating strategies [115].

Exercise is a well-known activator of mitochondrial biogenesis by upregulating PGC-1 $\alpha$ [116]. In addition to its effects on skeletal muscle, exercise training was shown to increase mitochondrial biogenesis in the brain [117], highlighting exercise as a complementary strategy for neuroprotection and management of mitochondrial-associated neurological disease [118].

Restoration of the mitochondrial fission-fusion equilibrium appeals as a therapeutic strategy for mitochondrial dysfunction, given the association between excessive mitochondrial fission and/or defective fusion in neurodegenerative disorders $[119,120]$. The hydrazone M1 was recently identified as a small molecule mitochondrial fusion promoter, capable of inhibiting mitochondrial fragmentation and enhancing survival of $\mathrm{MPP}^{+}$-treated SH-SY5Y neuroblastoma cells. M1 dose-dependently increased mitochondrial fusion in Mfn1 or Mfn2 single knockouts (KO), but not in Mfn1/2 double KO or OPA1 KO cells, suggesting it requires basal fusion activity, albeit the proposed mechanism of action is increased ATP5A/B expression levels [121]. Small molecule inhibitors of mitochondrial fission are also under investigation [122]. The selective Drp1 inhibitor mdivi-1 rescued abnormal mitochondrial dynamics induced by human mutant PINK1 in dopaminergic N27 neuronal cells [123], and was reported neuroprotective in rodent models with induced seizures [124] or ischemic injuries $[125,126]$. A novel peptide inhibitor of Drp1, P110, selectively inhibited Drp1 activation and mitochondrial Drp1-Fis1 interaction only under stressed conditions in cultured neuronal cells. P110 reduced mitochondrial fragmentation in SH-SY5Y cells treated with $\mathrm{MPP}^{+}$or mitochondrial uncoupler (CCCP), and reduced neurite loss in primary dopaminergic neurons challenged with $\mathrm{MPP}^{+}$to model PD neurodegeneration [127]. P110 was also reported to reduce mitochondrial fragmentation in HD cells, decreasing mitochondrial dysfunction, neuropathology and mortality in R6/2 HD mice [128].

\subsubsection{Movement and mitophagy}

Stimulating mitochondrial movement is a possible strategy to overcome deficits in mitochondrial distribution, particularly in neurons where mitochondria must travel extensively to meet the variable energy and $\mathrm{Ca}^{2+}$-buffering needs of distant synapses. AD and HD brain samples present decreased tubulin acetylation and cellular models of these diseases exhibit impaired microtubule-dependent mitochondrial transport [129-131]. Inhibition of the 
microtubule deacetylase HDAC6 with tubacin increased $\alpha$-tubulin acetylation, promoting motor recruitment and enhancing bidirectional transport in HD cellular models [130]. Another HDAC6 inhibitor, tubastatin A, rescued mitochondrial transport in amyloid- $\beta(A \beta)$ treated hippocampal neurons [131], as did HDAC6 deletion, which also improved memory function in $\mathrm{AD}$ mice without affecting $\mathrm{A} \beta$ plaque load [132]. HDAC6 inhibitors also corrected mitochondrial transport and reversed axonal loss in a mouse model of peripheral neuropathy [133]. The normally nuclear-located HDAC1 was suggested to play a critical role in axonal damage and abnormal mitochondrial transport, following neuroinflammatory stimuli (glutamate $+\mathrm{TNF} \alpha$ ) capable of evoking the $\mathrm{Ca}^{2+}$-dependent nuclear export of HDAC1 [134]. Further concerning $\mathrm{Ca}^{2+}$-dependent effects, pan-KDAC inhibitors were shown to improve neuronal $\mathrm{Ca}^{2+}$ recovery following glutamate receptor activation [135]. Moreover, HDAC1 inhibition with MS275, but not HDAC6 inhibition with tubacin, prevented cytosolic HDAC1 from disturbing mitochondrial transport following neuroinflammatory stimuli [134]. Available evidence therefore suggests that different KDACs may impair neuronal mitochondrial dynamics depending on the pathogenic insult. KDAC inhibition as a therapeutic strategy for rescuing mitochondrial transport must, nevertheless, be balanced against the possible interferences with other roles, namely that of HDAC6 in autophagy/mitophagy [109].

Mitophagy dysregulation, either defective or excessive, is associated with neurodegeneration [136]. Experiments in yeast suggest that mitophagy can be mechanistically separated from other forms of autophagy [137], prompting the search for selective mitophagy triggers in mammals. The PINK1/Parkin pathway appears to be a prominent player in mammalian mitophagy. Briefly, damaged mitochondria with collapsed $\Delta \psi_{\mathrm{m}}$ fail to import and process PINK1, which accumulates outside mitochondria recruiting the ubiquitin-ligase Parkin. Ubiquitin-binding adaptors (e.g. p62, HDAC6) recognize ubiquitinated mitochondria and facilitate the interaction with the autophagosomal protein LC3, igniting a cascade that culminates in autophagosome-lysosome fusion and mitochondrial digestion [138]. The postmitotic nature of neurons and their extreme morphology pose a unique challenge for mitochondrial turnover, being unlikely that neurons follow the same mitophagy rules as most mammalian cells. Interestingly, it was recently reported that at least some neurons shed axonal mitochondria for transcellular degradation by adjacent astrocytes [139]. Also, major bioenergetic differences between neurons and cell lines raise doubts on the relevance of the PINK1/Parkin pathway in neuronal mitophagy [140] [141], but other studies are supportive of that pathway in neurons [142, 143], where mitochondrial deubiquitinase USP30 was recently reported to oppose parkin-mediated mitophagy [144]. Currently, the (patho)-physiological triggers for neuronal mitophagy and the mechanisms for handling damaged mitochondria in distant synapses remain insufficiently understood [138], thus posing an outstanding challenge for developing mitophagy-related therapeutic strategies. Still, a study with heteroplasmic cybrid cells reported a selection against a deleterious mtDNA mutation by means of Parkin overexpression, thus suggesting that this strategy of enhancing mitophagy may ameliorate certain mitochondrial diseases [145].

\section{CONCLUDING REMARKS}

Currently available therapies for mitochondrial-associated neurological diseases are mostly palliative. Advances in understanding the molecular aetiology of mitochondrial dysfunction, together with breakthroughs in assisted conception, gene therapy and mitochondrial drug targeting strategies, fuel the expectation that effective preventive and disease-modifying therapeutics might be available in the near future. To achieve this goal, it is critical to develop 
good in vivo models for pre-clinical studies, organize patient cohorts and registries, establish reliable biomarkers, and improve clinical trial design. As always, and particularly in the case of complex mitochondrial disorders, clinical research will benefit tremendously from the unravelling of molecular pathogenesis afforded by basic mitochondrial research.

\section{ACKNOWLEDGMENTS}

Supported by Fundação para a Ciência e a Tecnologia (PEst-C/EQB/LA0006/2013; SFRH/BD/72071/2010; PTDC/NEU-NMC/0237/2012) and COMPETE/FEDER (FCOMP01-0124-FEDER-029649).

\section{AUTHOR CONTRIBUTIONS}

ALR performed the majority of literature search and wrote the first draft. BRP contributed to section 2.3. PGD contributed to section 2.4. JMAO coordinated the work and wrote the paper. All authors read and approved the final version.

\section{REFERENCES}

1. Kann O, Kovacs R. Mitochondria and neuronal activity. Am J Physiol Cell Physiol 2007; 292:C641-57.

2. Koopman WJ, Distelmaier F, Smeitink JA, Willems PH. OXPHOS mutations and neurodegeneration. EMBO J 2013; 32:9-29.

3. Tuppen HA, Blakely EL, Turnbull DM, Taylor RW. Mitochondrial DNA mutations and human disease. Biochim Biophys Acta 2010; 1797:113-28.

4. DiMauro S, Schon EA, Carelli V, Hirano M. The clinical maze of mitochondrial neurology. Nat Rev Neurol 2013; 9:429-44.

5. McFarland R, Taylor RW, Turnbull DM. A neurological perspective on mitochondrial disease. Lancet Neurol 2010; 9:829-40.

6. Schon EA, Przedborski S. Mitochondria: the next (neurode)generation. Neuron 2011; 70:1033-53.

7. Oliveira JM. Mitochondrial bioenergetics and dynamics in Huntington's disease: tripartite synapses and selective striatal degeneration. J Bioenerg Biomembr 2010; 42:227-34.

8. Oliveira JM, Goncalves J. In situ mitochondrial $\mathrm{Ca} 2+$ buffering differences of intact neurons and astrocytes from cortex and striatum. J Biol Chem 2009; 284:5010-20.

9. Pinto M, Pickrell AM, Moraes CT. Regional susceptibilities to mitochondrial dysfunctions in the CNS. Biol Chem 2012; 393:275-81.

10. Trounce IA, Pinkert CA. Cybrid models of mtDNA disease and transmission, from cells to mice. Curr Top Dev Biol 2007; 77:157-83.

11. Barrientos A. Yeast models of human mitochondrial diseases. IUBMB Life 2003; 55:8395.

12. Abramov AY, Smulders-Srinivasan TK, Kirby DM, Acin-Perez R, Enriquez JA, Lightowlers RN, et al. Mechanism of neurodegeneration of neurons with mitochondrial DNA mutations. Brain 2010; 133:797-807.

13. Oliveira JM. Techniques to investigate neuronal mitochondrial function and its pharmacological modulation. Curr Drug Targets 2011; 12:762-73. 
The published manuscript is available at EurekaSelect via http://www.eurekaselect.com/128349/article

14. Farrar GJ, Chadderton N, Kenna PF, Millington-Ward S. Mitochondrial disorders: aetiologies, models systems, and candidate therapies. Trends Genet 2013; 29:488-97.

15. Tyynismaa H, Suomalainen A. Mouse models of mitochondrial DNA defects and their relevance for human disease. EMBO Rep 2009; 10:137-43.

16. Steele SL, Prykhozhij SV, Berman JN. Zebrafish as a model system for mitochondrial biology and diseases: a review. Transl Res 2013.

17. Pinho BR, Santos MM, Fonseca-Silva A, Valentao P, Andrade PB, Oliveira JM. How mitochondrial dysfunction affects zebrafish development and cardiovascular function: an in vivo model for testing mitochondria-targeted drugs. Br J Pharmacol 2013; 169:1072-90.

18. Sanchez-Martinez A, Luo N, Clemente P, Adan C, Hernandez-Sierra R, Ochoa P, et al. Modeling human mitochondrial diseases in flies. Biochim Biophys Acta 2006; 1757:11908.

19. Ventura N, Rea SL. Caenorhabditis elegans mitochondrial mutants as an investigative tool to study human neurodegenerative diseases associated with mitochondrial dysfunction. Biotechnol J 2007; 2:584-95.

20. Pfeffer G, Horvath R, Klopstock T, Mootha VK, Suomalainen A, Koene S, et al. New treatments for mitochondrial disease-no time to drop our standards. Nat Rev Neurol 2013; 9:474-81.

21. Vento JM, Pappa B. Genetic counseling in mitochondrial disease. Neurotherapeutics 2013; 10:243-50.

22. Mitalipov S, Amato P, Parry S, Falk MJ. Limitations of preimplantation genetic diagnosis for mitochondrial DNA diseases. Cell Rep 2014; 7:935-7.

23. Craven L, Elson JL, Irving L, Tuppen HA, Lister LM, Greggains GD, et al. Mitochondrial DNA disease: new options for prevention. Hum Mol Genet 2011; 20:R168-74.

24. Amato P, Tachibana M, Sparman M, Mitalipov S. Three-parent in vitro fertilization: gene replacement for the prevention of inherited mitochondrial diseases. Fertil Steril 2014; 101:31-5.

25. Sato A, Kono T, Nakada K, Ishikawa K, Inoue S, Yonekawa H, et al. Gene therapy for progeny of mito-mice carrying pathogenic mtDNA by nuclear transplantation. Proc Natl Acad Sci U S A 2005; 102:16765-70.

26. Craven L, Tuppen HA, Greggains GD, Harbottle SJ, Murphy JL, Cree LM, et al. Pronuclear transfer in human embryos to prevent transmission of mitochondrial DNA disease. Nature 2010; 465:82-5.

27. Tachibana M, Sparman M, Sritanaudomchai H, Ma H, Clepper L, Woodward J, et al. Mitochondrial gene replacement in primate offspring and embryonic stem cells. Nature 2009; 461:367-72.

28. Tachibana M, Amato P, Sparman M, Woodward J, Sanchis DM, Ma H, et al. Towards germline gene therapy of inherited mitochondrial diseases. Nature 2013; 493:627-31.

29. Paull D, Emmanuele V, Weiss KA, Treff N, Stewart L, Hua H, et al. Nuclear genome transfer in human oocytes eliminates mitochondrial DNA variants. Nature 2013; 493:6327.

30. Chinnery PF, Craven L, Mitalipov S, Stewart JB, Herbert M, Turnbull DM. The challenges of mitochondrial replacement. PLoS Genet 2014; 10:e1004315.

31. Chinnery PF, Hudson G. Mitochondrial genetics. Br Med Bull 2013; 106:135-59.

32. Wang T, Sha H, Ji D, Zhang HL, Chen D, Cao Y, et al. Polar body genome transfer for preventing the transmission of inherited mitochondrial diseases. Cell 2014; 157:1591-604.

33. Wolf DP, Mitalipov S. Mitochondrial replacement therapies can circumvent mtDNA-based disease transmission. Cell Metab 2014; 20:6-8.

34. Moraes CT, Bacman SR, Williams SL. Manipulating mitochondrial genomes in the clinic: playing by different rules. Trends Cell Biol 2014; 24:209-11. 
The published manuscript is available at EurekaSelect via http://www.eurekaselect.com/128349/article

35. Smith PM, Lightowlers RN. Altering the balance between healthy and mutated mitochondrial DNA. J Inherit Metab Dis 2011; 34:309-13.

36. Kyriakouli DS, Boesch P, Taylor RW, Lightowlers RN. Progress and prospects: gene therapy for mitochondrial DNA disease. Gene Ther 2008; 15:1017-23.

37. Taylor RW, Chinnery PF, Turnbull DM, Lightowlers RN. Selective inhibition of mutant human mitochondrial DNA replication in vitro by peptide nucleic acids. Nat Genet 1997; 15:212-5.

38. Comte C, Tonin Y, Heckel-Mager AM, Boucheham A, Smirnov A, Aure K, et al. Mitochondrial targeting of recombinant RNAs modulates the level of a heteroplasmic mutation in human mitochondrial DNA associated with Kearns Sayre Syndrome. Nucleic Acids Res 2013; 41:418-33.

39. Tonin Y, Heckel AM, Dovydenko I, Meschaninova M, Comte C, Venyaminova A, et al. Characterization of chemically modified oligonucleotides targeting a pathogenic mutation in human mitochondrial DNA. Biochimie 2013.

40. Tonin Y, Heckel AM, Dovydenko I, Meschaninova M, Comte C, Venyaminova A, et al. Characterization of chemically modified oligonucleotides targeting a pathogenic mutation in human mitochondrial DNA. Biochimie 2014; 100:192-9.

41. Srivastava S, Moraes CT. Manipulating mitochondrial DNA heteroplasmy by a mitochondrially targeted restriction endonuclease. Hum Mol Genet 2001; 10:3093-9.

42. Tanaka M, Borgeld HJ, Zhang J, Muramatsu S, Gong JS, Yoneda M, et al. Gene therapy for mitochondrial disease by delivering restriction endonuclease SmaI into mitochondria. $\mathrm{J}$ Biomed Sci 2002; 9:534-41.

43. Bayona-Bafaluy MP, Blits B, Battersby BJ, Shoubridge EA, Moraes CT. Rapid directional shift of mitochondrial DNA heteroplasmy in animal tissues by a mitochondrially targeted restriction endonuclease. Proc Natl Acad Sci U S A 2005; 102:14392-7.

44. Bacman SR, Williams SL, Garcia S, Moraes CT. Organ-specific shifts in mtDNA heteroplasmy following systemic delivery of a mitochondria-targeted restriction endonuclease. Gene Ther 2010; 17:713-20.

45. Bacman SR, Williams SL, Duan D, Moraes CT. Manipulation of mtDNA heteroplasmy in all striated muscles of newborn mice by AAV9-mediated delivery of a mitochondriatargeted restriction endonuclease. Gene Ther 2012; 19:1101-6.

46. Bacman SR, Williams SL, Hernandez D, Moraes CT. Modulating mtDNA heteroplasmy by mitochondria-targeted restriction endonucleases in a 'differential multiple cleavage-site' model. Gene Ther 2007; 14:1309-18.

47. Minczuk M, Papworth MA, Miller JC, Murphy MP, Klug A. Development of a singlechain, quasi-dimeric zinc-finger nuclease for the selective degradation of mutated human mitochondrial DNA. Nucleic Acids Res 2008; 36:3926-38.

48. Minczuk M, Kolasinska-Zwierz P, Murphy MP, Papworth MA. Construction and testing of engineered zinc-finger proteins for sequence-specific modification of mtDNA. Nat Protoc 2010; 5:342-56.

49. Joung JK, Sander JD. TALENs: a widely applicable technology for targeted genome editing. Nat Rev Mol Cell Biol 2013; 14:49-55.

50. Bacman SR, Williams SL, Pinto M, Peralta S, Moraes CT. Specific elimination of mutant mitochondrial genomes in patient-derived cells by mitoTALENs. Nat Med 2013; 19:11113.

51. Gammage PA, Rorbach J, Vincent AI, Rebar EJ, Minczuk M. Mitochondrially targeted ZFNs for selective degradation of pathogenic mitochondrial genomes bearing large-scale deletions or point mutations. EMBO Mol Med 2014; 6:458-66.

52. Moraes CT. A magic bullet to specifically eliminate mutated mitochondrial genomes from patients' cells. EMBO Mol Med 2014; 6:434-5. 
The published manuscript is available at EurekaSelect via http://www.eurekaselect.com/128349/article

53. Nagley P, Farrell LB, Gearing DP, Nero D, Meltzer S, Devenish RJ. Assembly of functional proton-translocating ATPase complex in yeast mitochondria with cytoplasmically synthesized subunit 8 , a polypeptide normally encoded within the organelle. Proc Natl Acad Sci U S A 1988; 85:2091-5.

54. Perales-Clemente E, Fernandez-Silva P, Acin-Perez R, Perez-Martos A, Enriquez JA. Allotopic expression of mitochondrial-encoded genes in mammals: achieved goal, undemonstrated mechanism or impossible task? Nucleic Acids Res 2011; 39:225-34.

55. Manfredi G, Fu J, Ojaimi J, Sadlock JE, Kwong JQ, Guy J, et al. Rescue of a deficiency in ATP synthesis by transfer of MTATP6, a mitochondrial DNA-encoded gene, to the nucleus. Nat Genet 2002; 30:394-9.

56. Ojaimi J, Pan J, Santra S, Snell WJ, Schon EA. An algal nucleus-encoded subunit of mitochondrial ATP synthase rescues a defect in the analogous human mitochondrialencoded subunit. Mol Biol Cell 2002; 13:3836-44.

57. Bokori-Brown M, Holt IJ. Expression of algal nuclear ATP synthase subunit 6 in human cells results in protein targeting to mitochondria but no assembly into ATP synthase. Rejuvenation Res 2006; 9:455-69.

58. Guy J, Qi X, Pallotti F, Schon EA, Manfredi G, Carelli V, et al. Rescue of a mitochondrial deficiency causing Leber Hereditary Optic Neuropathy. Ann Neurol 2002; 52:534-42.

59. Oca-Cossio J, Kenyon L, Hao H, Moraes CT. Limitations of allotopic expression of mitochondrial genes in mammalian cells. Genetics 2003; 165:707-20.

60. Bonnet C, Kaltimbacher V, Ellouze S, Augustin S, Benit P, Forster V, et al. Allotopic mRNA localization to the mitochondrial surface rescues respiratory chain defects in fibroblasts harboring mitochondrial DNA mutations affecting complex I or v subunits. Rejuvenation Res 2007; 10:127-44.

61. Bonnet C, Augustin S, Ellouze S, Benit P, Bouaita A, Rustin P, et al. The optimized allotopic expression of ND1 or ND4 genes restores respiratory chain complex I activity in fibroblasts harboring mutations in these genes. Biochim Biophys Acta 2008; 1783:170717.

62. Ellouze S, Augustin S, Bouaita A, Bonnet C, Simonutti M, Forster V, et al. Optimized allotopic expression of the human mitochondrial ND4 prevents blindness in a rat model of mitochondrial dysfunction. Am J Hum Genet 2008; 83:373-87.

63. Lam BL, Feuer WJ, Abukhalil F, Porciatti V, Hauswirth WW, Guy J. Leber hereditary optic neuropathy gene therapy clinical trial recruitment: year 1. Arch Ophthalmol 2010; 128:1129-35.

64. Lam BL, Feuer WJ, Schiffman JC, Porciatti V, Vandenbroucke R, Rosa PR, et al. Trial end points and natural history in patients with G11778A Leber hereditary optic neuropathy : preparation for gene therapy clinical trial. JAMA Ophthalmol 2014; 132:428-36.

65. Koilkonda RD, Yu H, Chou TH, Feuer WJ, Ruggeri M, Porciatti V, et al. Safety and effects of the vector for the Leber hereditary optic neuropathy gene therapy clinical trial. JAMA Ophthalmol 2014; 132:409-20.

66. Kolesnikova OA, Entelis NS, Jacquin-Becker C, Goltzene F, Chrzanowska-Lightowlers ZM, Lightowlers RN, et al. Nuclear DNA-encoded tRNAs targeted into mitochondria can rescue a mitochondrial DNA mutation associated with the MERRF syndrome in cultured human cells. Hum Mol Genet 2004; 13:2519-34.

67. Rubio MA, Rinehart JJ, Krett B, Duvezin-Caubet S, Reichert AS, Soll D, et al. Mammalian mitochondria have the innate ability to import tRNAs by a mechanism distinct from protein import. Proc Natl Acad Sci U S A 2008; 105:9186-91.

68. Wang G, Shimada E, Zhang J, Hong JS, Smith GM, Teitell MA, et al. Correcting human mitochondrial mutations with targeted RNA import. Proc Natl Acad Sci U S A 2012; 109:4840-5. 
The published manuscript is available at EurekaSelect via http://www.eurekaselect.com/128349/article

69. Hornig-Do HT, Montanari A, Rozanska A, Tuppen HA, Almalki AA, Abg-Kamaludin DP, et al. Human mitochondrial leucyl tRNA synthetase can suppress non cognate pathogenic mt-tRNA mutations. EMBO Mol Med 2014; 6:183-93.

70. Ling J, Roy H, Qin D, Rubio MA, Alfonzo JD, Fredrick K, et al. Pathogenic mechanism of a human mitochondrial tRNAPhe mutation associated with myoclonic epilepsy with ragged red fibers syndrome. Proc Natl Acad Sci U S A 2007; 104:15299-304.

71. Rorbach J, Yusoff AA, Tuppen H, Abg-Kamaludin DP, Chrzanowska-Lightowlers ZM, Taylor RW, et al. Overexpression of human mitochondrial valyl tRNA synthetase can partially restore levels of cognate mt-tRNAVal carrying the pathogenic C25U mutation. Nucleic Acids Res 2008; 36:3065-74.

72. Park H, Davidson E, King MP. Overexpressed mitochondrial leucyl-tRNA synthetase suppresses the A3243G mutation in the mitochondrial tRNA(Leu(UUR)) gene. RNA 2008; 14:2407-16.

73. Li R, Guan MX. Human mitochondrial leucyl-tRNA synthetase corrects mitochondrial dysfunctions due to the tRNALeu(UUR) A3243G mutation, associated with mitochondrial encephalomyopathy, lactic acidosis, and stroke-like symptoms and diabetes. Mol Cell Biol 2010; 30:2147-54.

74. Karicheva OZ, Kolesnikova OA, Schirtz T, Vysokikh MY, Mager-Heckel AM, Lombes A, et al. Correction of the consequences of mitochondrial $3243 \mathrm{~A}>\mathrm{G}$ mutation in the MT-TL1 gene causing the MELAS syndrome by tRNA import into mitochondria. Nucleic Acids Res 2011; 39:8173-86.

75. Perli E, Giordano C, Pisano A, Montanari A, Campese AF, Reyes A, et al. The isolated carboxy-terminal domain of human mitochondrial leucyl-tRNA synthetase rescues the pathological phenotype of mitochondrial tRNA mutations in human cells. EMBO Mol Med 2014; 6:169-82.

76. Tyynismaa H, Schon EA. Mixing and matching mitochondrial aminoacyl synthetases and their tRNAs: a new way to treat respiratory chain disorders? EMBO Mol Med 2014; 6:155-7.

77. Hakkaart GA, Dassa EP, Jacobs HT, Rustin P. Allotopic expression of a mitochondrial alternative oxidase confers cyanide resistance to human cell respiration. EMBO Rep 2006; 7:341-5.

78. Dassa EP, Dufour E, Goncalves S, Jacobs HT, Rustin P. The alternative oxidase, a tool for compensating cytochrome c oxidase deficiency in human cells. Physiol Plant 2009; 137:427-34.

79. El-Khoury R, Dufour E, Rak M, Ramanantsoa N, Grandchamp N, Csaba Z, et al. Alternative oxidase expression in the mouse enables bypassing cytochrome c oxidase blockade and limits mitochondrial ROS overproduction. PLoS Genet 2013; 9:e1003182.

80. Bai Y, Hajek P, Chomyn A, Chan E, Seo BB, Matsuno-Yagi A, et al. Lack of complex I activity in human cells carrying a mutation in MtDNA-encoded ND4 subunit is corrected by the Saccharomyces cerevisiae NADH-quinone oxidoreductase (NDI1) gene. J Biol Chem 2001; 276:38808-13.

81. Seo BB, Nakamaru-Ogiso E, Flotte TR, Matsuno-Yagi A, Yagi T. In vivo complementation of complex I by the yeast Ndil enzyme. Possible application for treatment of Parkinson disease. J Biol Chem 2006; 281:14250-5.

82. Marella M, Seo BB, Nakamaru-Ogiso E, Greenamyre JT, Matsuno-Yagi A, Yagi T. Protection by the NDI1 gene against neurodegeneration in a rotenone rat model of Parkinson's disease. PLoS One 2008; 3:e1433.

83. Barber-Singh J, Seo BB, Nakamaru-Ogiso E, Lau YS, Matsuno-Yagi A, Yagi T. Neuroprotective effect of long-term NDI1 gene expression in a chronic mouse model of Parkinson disorder. Rejuvenation Res 2009; 12:259-67. 
The published manuscript is available at EurekaSelect via http://www.eurekaselect.com/128349/article

84. Marella M, Seo BB, Thomas BB, Matsuno-Yagi A, Yagi T. Successful amelioration of mitochondrial optic neuropathy using the yeast NDI1 gene in a rat animal model. PLoS One 2010; 5:e11472.

85. Chadderton N, Palfi A, Millington-Ward S, Gobbo O, Overlack N, Carrigan M, et al. Intravitreal delivery of AAV-NDI1 provides functional benefit in a murine model of Leber hereditary optic neuropathy. Eur J Hum Genet 2013; 21:62-8.

86. Vilain S, Esposito G, Haddad D, Schaap O, Dobreva MP, Vos M, et al. The yeast complex I equivalent NADH dehydrogenase rescues pink1 mutants. PLoS Genet 2012; 8:e1002456.

87. Smith RA, Hartley RC, Cocheme HM, Murphy MP. Mitochondrial pharmacology. Trends Pharmacol Sci 2012; 33:341-52.

88. Smith RA, Murphy MP. Animal and human studies with the mitochondria-targeted antioxidant MitoQ. Ann N Y Acad Sci 2010; 1201:96-103.

89. Miquel E, Cassina A, Martinez-Palma L, Souza JM, Bolatto C, Rodriguez-Bottero S, et al. Neuroprotective effects of the mitochondria-targeted antioxidant MitoQ in a model of inherited amyotrophic lateral sclerosis. Free Radic Biol Med 2014; 70:204-13.

90. Snow BJ, Rolfe FL, Lockhart MM, Frampton CM, O'Sullivan JD, Fung V, et al. A doubleblind, placebo-controlled study to assess the mitochondria-targeted antioxidant MitoQ as a disease-modifying therapy in Parkinson's disease. Mov Disord 2010; 25:1670-4.

91. Birk AV, Chao WM, Bracken C, Warren JD, Szeto HH. Targeting mitochondrial cardiolipin and the cytochrome c/cardiolipin complex to promote electron transport and optimize mitochondrial ATP synthesis. Br J Pharmacol 2014; 171:2017-28.

92. Szeto HH, Schiller PW. Novel therapies targeting inner mitochondrial membrane--from discovery to clinical development. Pharm Res 2011; 28:2669-79.

93. Petri S, Kiaei M, Damiano M, Hiller A, Wille E, Manfredi G, et al. Cell-permeable peptide antioxidants as a novel therapeutic approach in a mouse model of amyotrophic lateral sclerosis. J Neurochem 2006; 98:1141-8.

94. Yang L, Zhao K, Calingasan NY, Luo G, Szeto HH, Beal MF. Mitochondria targeted peptides protect against 1-methyl-4-phenyl-1,2,3,6-tetrahydropyridine neurotoxicity. Antioxid Redox Signal 2009; 11:2095-104.

95. Manczak M, Mao P, Calkins MJ, Cornea A, Reddy AP, Murphy MP, et al. Mitochondriatargeted antioxidants protect against amyloid-beta toxicity in Alzheimer's disease neurons. J Alzheimers Dis 2010; 20 Suppl 2:S609-31.

96. Siegel MP, Kruse SE, Percival JM, Goh J, White CC, Hopkins HC, et al. Mitochondrialtargeted peptide rapidly improves mitochondrial energetics and skeletal muscle performance in aged mice. Aging Cell 2013; 12:763-71.

97. Fink MP, Macias CA, Xiao J, Tyurina YY, Jiang J, Belikova N, et al. HemigramicidinTEMPO conjugates: novel mitochondria-targeted anti-oxidants. Biochem Pharmacol 2007; 74:801-9.

98. Xun Z, Rivera-Sanchez S, Ayala-Pena S, Lim J, Budworth H, Skoda EM, et al. Targeting of XJB-5-131 to mitochondria suppresses oxidative DNA damage and motor decline in a mouse model of Huntington's disease. Cell Rep 2012; 2:1137-42.

99. Battigelli A, Russier J, Venturelli E, Fabbro C, Petronilli V, Bernardi P, et al. Peptidebased carbon nanotubes for mitochondrial targeting. Nanoscale 2013; 5:9110-7.

100. Wenz T, Diaz F, Spiegelman BM, Moraes CT. Activation of the PPAR/PGC-1alpha pathway prevents a bioenergetic deficit and effectively improves a mitochondrial myopathy phenotype. Cell Metab 2008; 8:249-56.

101. Yatsuga S, Suomalainen A. Effect of bezafibrate treatment on late-onset mitochondrial myopathy in mice. Hum Mol Genet 2012; 21:526-35. 
The published manuscript is available at EurekaSelect via http://www.eurekaselect.com/128349/article

102. Viscomi C, Bottani E, Civiletto G, Cerutti R, Moggio M, Fagiolari G, et al. In vivo correction of COX deficiency by activation of the AMPK/PGC-1alpha axis. Cell Metab 2011; 14:80-90.

103. Johri A, Calingasan NY, Hennessey TM, Sharma A, Yang L, Wille E, et al. Pharmacologic activation of mitochondrial biogenesis exerts widespread beneficial effects in a transgenic mouse model of Huntington's disease. Hum Mol Genet 2012; 21:1124-37.

104. Dumont M, Stack C, Elipenahli C, Jainuddin S, Gerges M, Starkova N, et al. Bezafibrate administration improves behavioral deficits and tau pathology in P301S mice. Hum Mol Genet 2012; 21:5091-105.

105. Noe N, Dillon L, Lellek V, Diaz F, Hida A, Moraes CT, et al. Bezafibrate improves mitochondrial function in the CNS of a mouse model of mitochondrial encephalopathy. Mitochondrion 2013; 13:417-26.

106. Miller BW, Willett KC, Desilets AR. Rosiglitazone and pioglitazone for the treatment of Alzheimer's disease. Ann Pharmacother 2011; 45:1416-24.

107. Carta AR. PPAR-gamma: therapeutic prospects in Parkinson's disease. Curr Drug Targets 2013; 14:743-51.

108. Wilkins HM, Harris JL, Carl SM, E L, Lu J, Eva Selfridge J, et al. Oxaloacetate activates brain mitochondrial biogenesis, enhances the insulin pathway, reduces inflammation and stimulates neurogenesis. Hum Mol Genet 2014.

109. Guedes-Dias P, Oliveira JM. Lysine deacetylases and mitochondrial dynamics in neurodegeneration. Biochim Biophys Acta 2013; 1832:1345-59.

110. Brenmoehl J, Hoeflich A. Dual control of mitochondrial biogenesis by sirtuin 1 and sirtuin 3. Mitochondrion 2013.

111. Gong B, Pan Y, Vempati P, Zhao W, Knable L, Ho L, et al. Nicotinamide riboside restores cognition through an upregulation of proliferator-activated receptor-gamma coactivator 1alpha regulated beta-secretase 1 degradation and mitochondrial gene expression in Alzheimer's mouse models. Neurobiol Aging 2013; 34:1581-8.

112. Pirinen E, Canto C, Jo YS, Morato L, Zhang H, Menzies KJ, et al. Pharmacological Inhibition of poly(ADP-ribose) polymerases improves fitness and mitochondrial function in skeletal muscle. Cell Metab 2014; 19:1034-41.

113. Khan NA, Auranen M, Paetau I, Pirinen E, Euro L, Forsstrom S, et al. Effective treatment of mitochondrial myopathy by nicotinamide riboside, a vitamin B3. EMBO Mol Med 2014; 6:721-31.

114. Cerutti R, Pirinen E, Lamperti C, Marchet S, Sauve AA, Li W, et al. NAD(+)dependent activation of Sirtl corrects the phenotype in a mouse model of mitochondrial disease. Cell Metab 2014; 19:1042-9.

115. Lightowlers RN, Chrzanowska-Lightowlers ZM. Salvaging hope: Is increasing NAD(+) a key to treating mitochondrial myopathy? EMBO Mol Med 2014; 6:705-7.

116. Lira VA, Benton CR, Yan Z, Bonen A. PGC-1alpha regulation by exercise training and its influences on muscle function and insulin sensitivity. Am J Physiol Endocrinol Metab 2010; 299:E145-61.

117. Steiner JL, Murphy EA, McClellan JL, Carmichael MD, Davis JM. Exercise training increases mitochondrial biogenesis in the brain. J Appl Physiol (1985) 2011; 111:1066-71.

118. Zhang Q, Wu Y, Zhang P, Sha H, Jia J, Hu Y, et al. Exercise induces mitochondrial biogenesis after brain ischemia in rats. Neuroscience 2012; 205:10-7.

119. Wang X, Su B, Lee HG, Li X, Perry G, Smith MA, et al. Impaired balance of mitochondrial fission and fusion in Alzheimer's disease. J Neurosci 2009; 29:9090-103.

120. Chen H, Chan DC. Mitochondrial dynamics--fusion, fission, movement, and mitophagy--in neurodegenerative diseases. Hum Mol Genet 2009; 18:R169-76. 
The published manuscript is available at EurekaSelect via http://www.eurekaselect.com/128349/article

121. Wang D, Wang J, Bonamy GM, Meeusen S, Brusch RG, Turk C, et al. A small molecule promotes mitochondrial fusion in mammalian cells. Angew Chem Int Ed Engl 2012; 51:9302-5.

122. Lackner LL, Nunnari J. Small molecule inhibitors of mitochondrial division: tools that translate basic biological research into medicine. Chem Biol 2010; 17:578-83.

123. Cui M, Tang X, Christian WV, Yoon Y, Tieu K. Perturbations in mitochondrial dynamics induced by human mutant PINK1 can be rescued by the mitochondrial division inhibitor mdivi-1. J Biol Chem 2010; 285:11740-52.

124. Xie N, Wang C, Lian Y, Zhang H, Wu C, Zhang Q. A selective inhibitor of Drp1, mdivi-1, protects against cell death of hippocampal neurons in pilocarpine-induced seizures in rats. Neurosci Lett 2013; 545:64-8.

125. Park SW, Kim KY, Lindsey JD, Dai Y, Heo H, Nguyen DH, et al. A selective inhibitor of drp1, mdivi-1, increases retinal ganglion cell survival in acute ischemic mouse retina. Invest Ophthalmol Vis Sci 2011; 52:2837-43.

126. Zhang N, Wang S, Li Y, Che L, Zhao Q. A selective inhibitor of Drp1, mdivi-1, acts against cerebral ischemia/reperfusion injury via an anti-apoptotic pathway in rats. Neurosci Lett 2013; 535:104-9.

127. Qi X, Qvit N, Su YC, Mochly-Rosen D. A novel Drp1 inhibitor diminishes aberrant mitochondrial fission and neurotoxicity. J Cell Sci 2013; 126:789-802.

128. Guo X, Disatnik MH, Monbureau M, Shamloo M, Mochly-Rosen D, Qi X. Inhibition of mitochondrial fragmentation diminishes Huntington's disease-associated neurodegeneration. J Clin Invest 2013; 123:5371-88.

129. Hempen B, Brion JP. Reduction of acetylated alpha-tubulin immunoreactivity in neurofibrillary tangle-bearing neurons in Alzheimer's disease. J Neuropathol Exp Neurol 1996; 55:964-72.

130. Dompierre JP, Godin JD, Charrin BC, Cordelieres FP, King SJ, Humbert S, et al. Histone deacetylase 6 inhibition compensates for the transport deficit in Huntington's disease by increasing tubulin acetylation. J Neurosci 2007; 27:3571-83.

131. Kim C, Choi H, Jung ES, Lee W, Oh S, Jeon NL, et al. HDAC6 inhibitor blocks amyloid beta-induced impairment of mitochondrial transport in hippocampal neurons. PLoS One 2012; 7:e42983.

132. Govindarajan N, Rao P, Burkhardt S, Sananbenesi F, Schluter OM, Bradke F, et al. Reducing HDAC6 ameliorates cognitive deficits in a mouse model for Alzheimer's disease. EMBO Mol Med 2013; 5:52-63.

133. d'Ydewalle C, Krishnan J, Chiheb DM, Van Damme P, Irobi J, Kozikowski AP, et al. HDAC6 inhibitors reverse axonal loss in a mouse model of mutant HSPB1-induced Charcot-Marie-Tooth disease. Nat Med 2011; 17:968-74.

134. Kim JY, Shen S, Dietz K, He Y, Howell O, Reynolds R, et al. HDAC1 nuclear export induced by pathological conditions is essential for the onset of axonal damage. Nat Neurosci 2010; 13:180-9.

135. Oliveira JM, Chen S, Almeida S, Riley R, Goncalves J, Oliveira CR, et al. Mitochondrial-dependent $\mathrm{Ca} 2+$ handling in Huntington's disease striatal cells: effect of histone deacetylase inhibitors. J Neurosci 2006; 26:11174-86.

136. Batlevi Y, La Spada AR. Mitochondrial autophagy in neural function, neurodegenerative disease, neuron cell death, and aging. Neurobiol Dis 2011; 43:46-51.

137. Kanki T, Klionsky DJ. Mitophagy in yeast occurs through a selective mechanism. J Biol Chem 2008; 283:32386-93.

138. Ashrafi G, Schwarz TL. The pathways of mitophagy for quality control and clearance of mitochondria. Cell Death Differ 2013; 20:31-42. 
The published manuscript is available at EurekaSelect via http://www.eurekaselect.com/128349/article

139. Davis CH, Kim KY, Bushong EA, Mills EA, Boassa D, Shih T, et al. Transcellular degradation of axonal mitochondria. Proc Natl Acad Sci U S A 2014; 111:9633-8.

140. Van Laar VS, Arnold B, Cassady SJ, Chu CT, Burton EA, Berman SB. Bioenergetics of neurons inhibit the translocation response of Parkin following rapid mitochondrial depolarization. Hum Mol Genet 2011; 20:927-40.

141. Grenier K, McLelland GL, Fon EA. Parkin- and PINK1-Dependent Mitophagy in Neurons: Will the Real Pathway Please Stand Up? Front Neurol 2013; 4:100.

142. Wang X, Winter D, Ashrafi G, Schlehe J, Wong YL, Selkoe D, et al. PINK1 and Parkin target Miro for phosphorylation and degradation to arrest mitochondrial motility. Cell 2011; 147:893-906.

143. Cai Q, Zakaria HM, Simone A, Sheng ZH. Spatial parkin translocation and degradation of damaged mitochondria via mitophagy in live cortical neurons. Curr Biol 2012; 22:54552 .

144. Bingol B, Tea JS, Phu L, Reichelt M, Bakalarski CE, Song Q, et al. The mitochondrial deubiquitinase USP30 opposes parkin-mediated mitophagy. Nature 2014; 510:370-5.

145. Suen DF, Narendra DP, Tanaka A, Manfredi G, Youle RJ. Parkin overexpression selects against a deleterious mtDNA mutation in heteroplasmic cybrid cells. Proc Natl Acad Sci U S A 2010; 107:11835-40. 